\title{
Rapid crack propagation in loosely fitted PE liner pipe
}

\section{P. Leevers*, A. Henderson and R. Pereira}

\begin{abstract}
Laboratory scale tests have been used to assess whether a loosely fitted PE liner within a rigid host pipe could, at any realistic working pressure, fail by rapid crack propagation (RCP). The standard 'S4' test method for RCP was modified, the usual cage of containment rings being replaced by a rigid, closed sleeve maintaining a predefined radial clearance from the unpressurised pipe. Pipe specimens of $125 \mathrm{~mm}$ diameter with wall thicknesses of 11.4 and $7.1 \mathrm{~mm}$, made from a PE80 polyethylene in which RCP is normally possible at $0^{\circ} \mathrm{C}$, were tested with three different clearances as well as with the usual S4 cage. For pipe having an unpressurised clearance down to $2 \%$ or so of the diameter, the RCP critical pressure was increased by $\sim 50 \%$ for both thicknesses; for smaller clearances, by progressively more. These results reinforce suspicions that pipe installation conditions may affect full scale RCP resistance.
\end{abstract}

Keywords: Polyethylene, Pipe, Liner, Rehabilitation, Gas, S4 test

\section{Introduction}

Several technologies are available for the rehabilitation of an existing, damaged pipeline by insertion of a plastics liner. In many cases, the existing pipeline has lost its ability to maintain pressure but retains much of its rigidity and strength. If so, the host pipe and the inserted liner share the two functions normally served by a single wall: preventing leakage and withstanding circumferential stress to maintain flow area. In this respect, lined pipes have enhanced structural reliability, as long as the liner material has the properties required of standard, self-supporting, single wall pipe. To satisfy this proviso, the liner material usually chosen is a designated pipe grade polyethylene (PE), whose resistance to long term pressure, to notches and to brittle rapid crack propagation ( $\mathrm{RCP}$ ) is already established. These materials are also known for their extraordinary ductility and some slip lining installation technologies exploit this to the full.

Pressurised pipe liner is under constant stress and must resist slow crack growth. To what extent, however, can (or need) a pipe liner be relied on to resist RCP? $\mathrm{RCP}$ has always been an extremely rare failure mode and it has become more so as design and test procedures to avoid it have become established. There is no record that RCP has ever been observed in liner pipe and this must remain the case.

The crack driving force $G$ for RCP is supplied in part by the release of hoop strain energy stored in the pipe wall ahead of the crack and in part by the direct forward force developed by pressure acting on the 'flared' pipe

Department of Mechanical Engineering, Imperial College London, South Kensington Campus, London SW7 2AZ, UK

*Corresponding author, email p.leevers@imperial.ac.uk wall behind the crack. The total crack driving force is resisted by the fracture resistance $G_{\mathrm{D}}$ of the pipe material, itself a difficult material property to evaluate. ${ }^{1}$ During steady RCP, $G=G_{\mathrm{D}}$. Solution of this equation would provide a prediction for the critical pressure $p_{\mathrm{c}}$, above which RCP can be sustained. Both $G$ and $G_{\mathrm{D}}$ depend on crack speed and on thickness. The dependence of $G_{\mathrm{D}}$ on thickness will be especially beneficial for liner, which is usually thinner than standard pipe.

Despite many analytical and computational attempts to solve this complex problem, e.g. Williams and Venizelos, ${ }^{2}$ none has yet achieved the broad acceptance and the extensive verification needed to predict critical pressure reliably, so that $p_{\mathrm{c}}$ must still be evaluated experimentally for each material and pipe size. In the 'Discussion' section below, a very simple model, developed by the authors and refined by Zhuang ${ }^{3}$ for comparison with a more elaborate finite element model, is used to explore the analogous case of a pipe buried in backfill, but it cannot be applied to the host liner case.

If a plastics liner is in contact with the rigid host pipe, both of the components which contribute to the driving force $G$ are reduced: the host pipe shares the hoop stress, while flaring is prevented. Thus, the critical pressure must increase by some factor which depends on the tightness of fit.

The simplest case to analyse is that in which the pressurised liner just makes contact with the host pipe, without loading it. If RCP occurs, this case becomes very similar to that of a pipe pressurised by an approximately incompressible fluid such as water: there is no flaring, but the full strain energy of the liner wall is available to drive the crack. For this case, Irwin and Corten derived (in a much quoted but unpublished report ${ }^{4}$ ) a solution for $G$ which when equated to $G_{\mathrm{D}}$, gives an expression for the critical pressure above which RCP can be sustained 


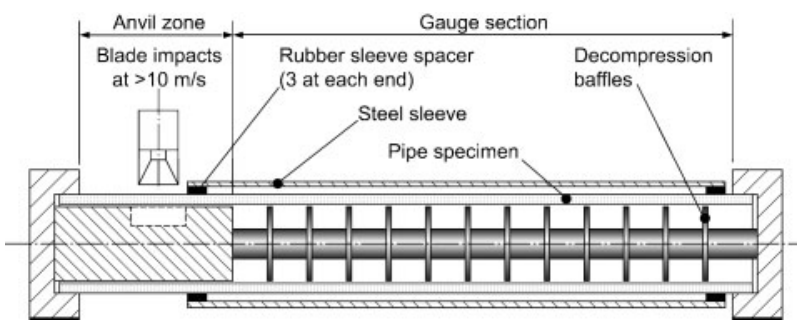

1 ISO 13477 'S4' test rig, modified to test effect of rigid containment

$$
p_{\mathrm{c}}=\frac{1}{D^{*}}\left(\frac{8 E G_{\mathrm{D}}}{\pi D}\right)^{1 / 2}
$$

Here, $E$ is the dynamic tensile modulus of the pipe wall material, $D$ the nominal outside pipe diameter and $D^{*}$ its ratio to the wall thickness, i.e. the standard dimensional ratio (SDR). The validity of this equation for water pipe and its limitations were studied in some detail by Greenshields and Leevers. ${ }^{5}$

The case of zero contact pressure is of interest only for reference. Even though initially achievable, this condition would be lost through changes in pressure, temperature and time.

The intermediate case, where a small gap exists between the liner and the host, is more complex. On the one hand, one might expect leakage of fluid forward into the annular gap to reduce the pressure difference across the liner pipe wall and thus, to reduce the flaring component; on the other, the annular gap would severely restrict the forward mass flowrate from a crack advancing at a typical speed of $100 \mathrm{~m} \mathrm{~s}^{-1}$. On the one hand, the physical restriction on flaring imposed by contact with the host pipe wall must reduce the flaring contribution to $G$; on the other, much of the work done in flaring is in fact stored in the elastic energy of outward 'ring bending' of the wall. Venizelos et al. ${ }^{6}$ found the effect on S4 critical pressure of constraint by gravel backfill to be significant: with increasing depth and compaction, the critical pressure of SDR 11 pipe was increased from 1.65 to 2.6 bar and for SDR 17 pipe, from 1.9 to 2.6 bar.

A well established, user friendly model of the RCP process would provide much needed assistance in judging the balance of these opposing effects. In the absence (or the expectation) of such a model, the authors turn to experiment.

\section{Experimental}

The effect of a containment sleeve on RCP critical pressure was investigated using a modified ISO 13477 'S4' test method for $125 \mathrm{~mm}$ diameter pipe. Each S4 test involves the initiation of an axial impact crack, and its propagation to a diameter or so in length, from near one end of a $7 D$ long pipe specimen. If the pipe is unpressurised, the crack soon arrests, but if the internal gas (air) pressure exceeds some critical value $p_{\mathrm{cS} 4}$, its internal energy takes over from that of the striker and can continue to drive the crack steadily along the remainder of the pipe. Internal baffles to suppress axial backflow and an external cage of concentric rings to prevent excessive flaring maintain a steady state within this 'gauge section' (Fig. 1). As a result, the crack

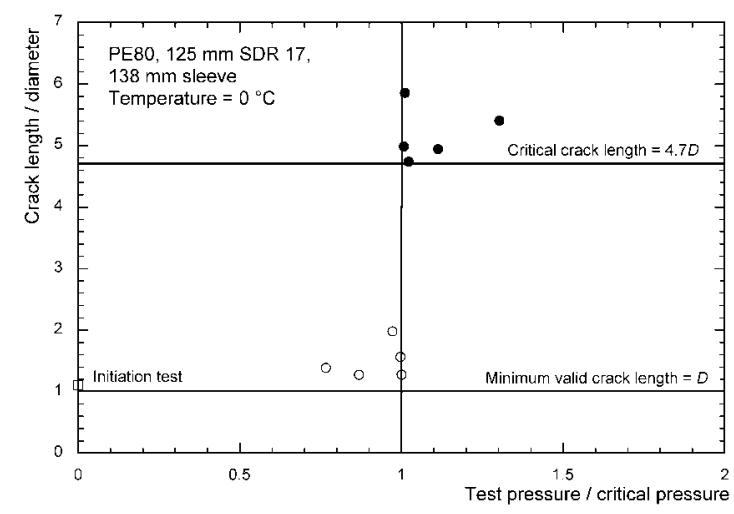

2 Individual test series (125 mm SDR17, $138 \mathrm{~mm}$ sleeve): open circles - arrest results; full circles - propagation results

seldom arrests within this region of pipe: it either arrests very soon after initiation, or propagates very nearly to the end.

Figure 1 shows the arrangement used for sleeved S4 tests. For baseline measurements of critical pressure to ISO 13477, this S4 apparatus was used in its standard form, the sleeve shown being replaced by a cage of containment rings. This cage presents little resistance to the outflow of pressurising air, but restricts flaring of the pipe to within a coaxial cylinder of diameter $1 \cdot 1 D$. The sleeve for liner simulation was made by rolling from $3 \mathrm{~mm}$ mild steel and butt welding along the axial seam. Three sleeves were produced, with internal diameters which ranged from $127 \mathrm{~mm}$, the minimum estimated to avoid contact by a $125 \mathrm{~mm}$ PE80 pipe expanded at maximum test pressure, to $138 \mathrm{~mm}$, the nominal internal dimension of a standard $1 \cdot 1 D$ diameter $\mathrm{S} 4$ test containment cage (to $\pm 1 \mathrm{~mm}$ precision).

Some attention and experimentation was devoted to how adequate concentricity of the cage should be maintained without affecting either the temperature of the pipe or its deformation during RCP. A simple solution was adopted. The sleeve was assembled onto the pipe before temperature conditioning, using three rubber spacers disposed at $120^{\circ}$ intervals around a circumference near each end. Spacers were built up to a thickness appropriate to each individual test. At the initiation end, these spacers are sufficiently close to the impact point for the striker to drive a crack between them, while at the far end they are sufficiently close to the end cap not to add significantly to its own external constraint. Care was taken to leave a small axial gap between the end of the sleeve and the end cap, so that steady state crack propagation should not be untypically disturbed by transient pressurisation of the outside pipe surface by forward outflow. After installation on the rig, the sleeve was supported near its centre by a car jack. Although out of roundness of the rolled pipe was apparent, especially near the weld, care was taken to test specimens with a consistent circumferential orientation so that the sleeve sector near the crack path was of uniform radius. These precautions reduced the clearance uncertainty in the crack region to an estimated $\pm 0.5 \mathrm{~mm}$.

All pipe specimens were notched internally in the initiation region, as permitted by ISO 13477. Each specimen-sleeve assembly was conditioned at $-2^{\circ} \mathrm{C}$ for at least $16 \mathrm{~h}$ and tested within the standard time limits so 


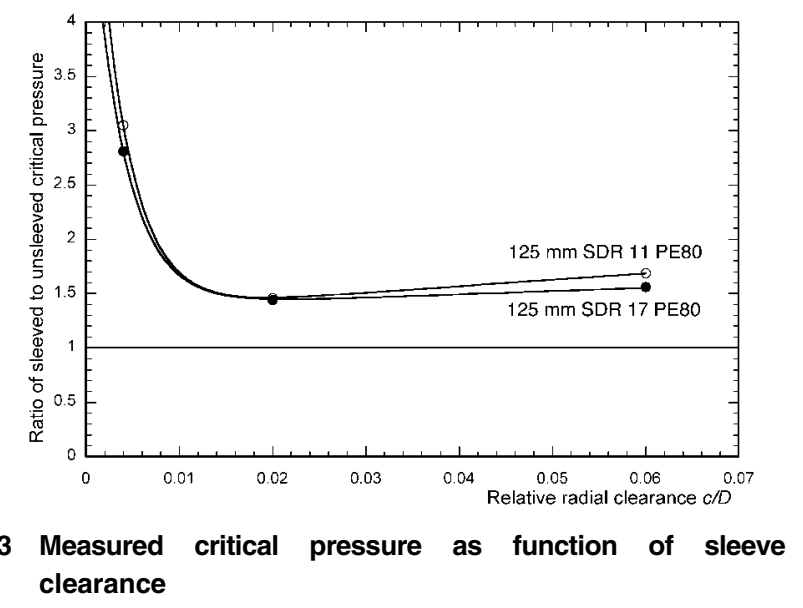

that the nominal test temperature of $0^{\circ} \mathrm{C}$ was maintained to within a consistent tolerance.

\section{Results}

The result of an individual S4 test is recorded as a final crack length from the initiation impact point, $v$. gauge pressure. A crack length of $4 \cdot 7 \mathrm{D}$ or more implies that the crack would continue to extend indefinitely if the specimen were longer, and is recorded as a 'propagation' result. A shorter crack is recorded as an 'arrest'.

At a nominal test temperature of $0^{\circ} \mathrm{C}$, this PE80 material provided the clear results hoped for, without indecisive arrests within the propagation zone of the kind recently discussed by Kosari et al. ${ }^{7}$ Not only the standard S4 method but also the 'sleeved' configurations gave well defined critical pressure results (Table 1). Figure 2 shows a standard S4 data plot of individual results for one such combination: SDR 17 pipe with a loose sleeve.

Figure 3 summarises all of the critical pressure results in a more generalised form: the critical pressure for each sleeved configuration is normalised against the critical pressure $p_{\mathrm{cS} 4}$ for the standard $\mathrm{S} 4$ configuration and plotted as a function of mean nominal sleeve clearance $c$ in the unpressurised state. Each sleeve now appears to have had virtually the same relative effect on critical pressure in $125 \mathrm{~mm}$ pipe of both SDRs (thicknesses). The results for each constraint condition showed good consistence, although the roundness of the steel containment sleeves left much to be desired and all dimensions quoted (including pipe dimensions) are nominal.

It was argued in the 'Introduction' that the IrwinCorten equation (1) should give a reasonable estimate of the critical pressure of a pipe in a sleeve without contact, but with zero clearance; this would, of course, be virtually impossible to achieve experimentally. Using the values of dynamic tensile modulus $E=2.5 \mathrm{GPa}$ and RCP resistance $G_{\mathrm{D}}=2 \cdot 5 \mathrm{~kJ} \mathrm{~m}^{-2}$ confirmed by the earlier work of Venizelos and Greenshields, ${ }^{6}$ the authors obtain the values $10 \cdot 3$ bar for SDR 11 and 6.64 bar for SDR 17. These values are respectively 6.64 and 5.72 times greater than those measured experimentally for air pressurisation in the $\mathrm{S} 4$ test. Because $G=G_{\mathrm{D}}$ during $\mathrm{RCP}$, these ratios are squared to arrive at a factor by which the driving force $G$ is underestimated by equation (1): 44 for SDR11 and 33 for SDR 17. This calculation thus gives some idea of how much the flaring action of gas pressure on the crack flaps contributes to the crack driving force and how carefully one must formulate a model (or even a discussion) of its effects.

These Irwin-Corten estimates are first used to fix the curve fits (exponential decay towards a constant positive gradient) shown in Fig. 3. These fits are not fully justified by the data, but the slight increase in critical pressure as relative cage clearance increases above $\sim 0.02$ is clearly seen in both sets of results.

\section{Discussion}

First, it is clear that RCP is possible in liner pipe as long as the liner remains 'loose' under pressure. The persistence of stable RCP suggests strongly that forward leakage of pressurising fluid through the liner/sleeve gap does not exert a significant crack closing force. If this were not so, progressive decrease of the pressure difference across the liner wall would be expected to generate 'short arrest' results in the region near the critical pressure and these are not seen. Evidently, the crack propagation velocity is high enough to outrun fluid outflow. However, the modest increase in critical pressure suggests that outflow does have some constraining effect on flaring behind the crack front.

It is important to ask how applicable these results are to 'full scale' conditions. Because service RCP failures remain virtually unknown, this term is taken to refer to another standard experimental configuration: that of the ISO 13478 full scale (FS) test. Here, the pipe specimen is buried in a temperature controlled trench and is constrained only by lightly compacted backfill at a depth much smaller than that used in installation. Crucially, the pipe specimen is much longer and does not contain the baffles which prevent the $\mathrm{S} 4$ specimen from decompressing before the crack arrives there. As a result, while the critical pressure measured using each of the two tests is very consistent, the two values ( $p_{\mathrm{cS} 4}$ and $\left.p_{\mathrm{cFS}}\right)$ are very different: the FS value is much higher.

The most widely accepted equation for correlating the two results, which gives a suitably conservative representation of available data and is written into ISO 13477 , is

$$
p_{\mathrm{cFS}}=3 \cdot 6 p_{\mathrm{cS} 4}+2 \cdot 6 \quad \text { (bar) }
$$

Equation (2) is derived from one-dimensional gas flow analysis ${ }^{8}$ using four main assumptions:

(i) everywhere ahead of the crack in the FS configuration, there is axial free backflow of gas

Table 1 Critical pressure results for standard ISO 13477 and sleeved S4 pipe tests

Critical pressure $p_{\mathrm{cS} 4}$ (gauge), bar

\begin{tabular}{lllll}
\cline { 2 - 4 } SDR & Standard S4 test cage & Tight sleeve $(c / D \sim 0.004)$ & Medium sleeve $(c / D=0.02)$ & Loose sleeve $(c / D=0 \cdot 06)$ \\
\hline 11 & 1.16 & 3.54 & 1.90 & 1.96 \\
17 & 1.55 & 4.36 & 2.23 & 2.42 \\
\hline
\end{tabular}




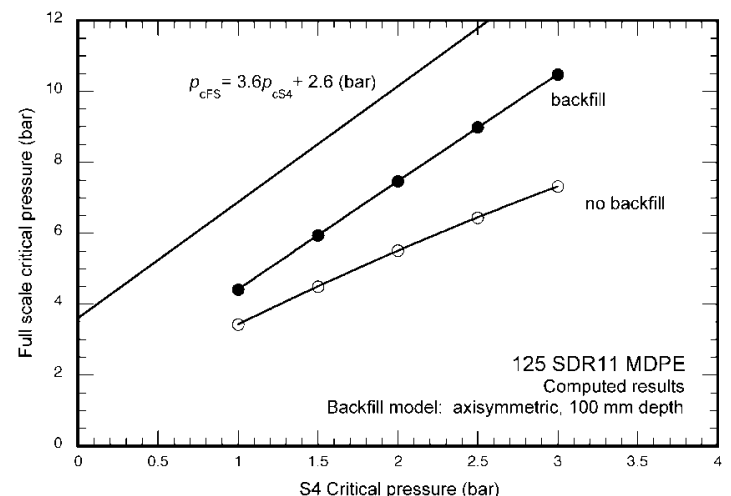

4 Computed full scale critical pressure with and without backfill simulation

(ii) everywhere ahead of the crack in the S4 test, the internal pressure remains at its initial value

(iii) immediately behind the crack front in either configuration, there is free outflow of the contained gas

(iv) immediately before a crack arrests at the FS critical pressure, it is propagating at a constant speed only just greater than zero.

Clearly, assumption (iii) is difficult to justify for a pipe in a gas tight containment. Assumption (iv) is, in fact, very difficult to justify for any polyethylene pipe material, or indeed for most thermoplastics; it is known that the resistance of polyethylene to cracks running at speeds lower than several tens of $\mathrm{m} \mathrm{s}^{-1}$ is very high indeed, so that fracture test methods cannot drive such a crack $^{1}$ and an arresting crack in a pipe appears to stop almost instantaneously. ${ }^{9}$

If assumption (iv) is invalid, the backflow analysis cannot fully explain the magnitude of observed $p_{\mathrm{cFS}} / p_{\mathrm{cS} 4}$ ratios (typically 5 or greater). Instead, the simple, analytical model of pipe fracture identified earlier, used alongside the usual one-dimensional gas analysis, confirms what Venizelos' experimental results had already indicated: ${ }^{6}$ that a significant proportion of the full scale critical pressure is generated by the resistance of the backfill.

Figure 4 plots the $p_{\mathrm{cFS}}$ versus $p_{\mathrm{cS} 4}$ relationship computed using the analytical model referred to in the 'Introduction'. The water saturated backfill is represented simply as a fluid of density $2200 \mathrm{~kg} \mathrm{~m}^{-3}$ which blankets the pipe axisymmetrically, to a thickness of $100 \mathrm{~mm}$. Each point is computed by computing both critical pressures from pipe wall toughness, which as treated as a dummy parameter, but has reassuringly realistic values $\left(\sim 5 \mathrm{~kJ} \mathrm{~m}^{-2}\right)$ for commonly measured critical pressures. Complete neglect of the backfill yields a seriously underestimated FS critical pressure, but some realism is restored by accounting only for its inertial resistance; a more sophisticated model would also account for the frictional forces need to deform it and for the hydrostatic pressure exerted by its weight.

One is led to suspect that the standard model for $p_{\mathrm{cFS}} /$ $p_{\text {cS4 }}$ correlation may yield good results largely by coincidence and that a full scale RCP test result may depend more strongly than expected on the backfill. The results of the present study add further emphasis to the need for a broad study of installation effects on full scale critical pressure measurements.

\section{Conclusions}

Experiments on SDR 11 and SDR 17 PE80 pipes of nominal diameter $D=125 \mathrm{~mm}$, using a modified S4 test method, indicate that rapid crack propagation is possible in a PE liner within a closed host pipe, as long as there is a radial clearance of at least $0.02 D$ before the liner is pressurised. However, the effect of the host pipe is to increase the critical pressure by at least $50 \%$. Caution is needed in extrapolating these results to the full scale case, and the influence of the backfill and other installation conditions on full scale critical pressures needs considerably more investigation.

\section{Acknowledgements}

The authors gratefully acknowledge the support of GPS, INEOS Polyolefins and Subterra for this work. This paper is based on a presentation at Plastics Pipes XIII held in Washington, DC in October 2006.

\section{References}

1. M. A. Wheel and P. S. Leevers: Int. J. Fract., 1993, 61, 331-348.

2. J. G. Williams and G. P. Venizelos: Int. J. Fract., 1998, 94, 161176

3. Z. Zhuang: 'The development of finite element methods for the investigation of dynamic crack propagation in gas pipelines', $\mathrm{PhD}$ thesis, University College Dublin, Dublin, Ireland, 1995.

4. G. R. Irwin and H. T. Corten: Report to Northern Natural Gas Co. and El Paso Natural Gas Co., 1968.

5. C. J. Greenshields and P. S. Leevers: Int. J. Fract., 1996, 79, 85-95.

6. G. P. Venizelos, C. J. Greenshields, A. Ivankovic and P. S. Leevers: Plast. Rubber Compos. Process. Appl., 1997, 26, $394-403$.

7. T. Kosario, K. Oliphant, P. Vibien and P. S. Leevers: Proc. 13th Int. Conf. on 'Plastics pipes', Washington, DC, USA, October 2006, Paper 4, Session 6b.

8. C. J. Greenshields and P. S. Leevers: Plast. Rubber Compos. Process. Appl., 1999, 28, 20-25.

9. P. Yayla and P. S. Leevers: Eng. Fract. Mech., 1992, 42, 675-682. 